\title{
Radiative natural SUSY spectrum from deflected AMSB scenario with messenger-matter interactions
}

\author{
Fei Wang, ${ }^{a, b}$ Jin Min Yang ${ }^{b, c}$ and Yang Zhang ${ }^{b}$ \\ ${ }^{a}$ School of Physics, Zhengzhou University, \\ Zhengzhou 450000, P.R. China \\ ${ }^{b}$ State Key Laboratory of Theoretical Physics, \\ Institute of Theoretical Physics, Chinese Academy of Sciences, \\ Beijing 100080, P.R. China \\ ${ }^{c}$ Department of Physics, Tohoku University, \\ Sendai 980-8578, Japan \\ E-mail: feiwang@zzu.edu.cn, jmyang@itp.ac.cn, zhangyang@itp.ac.cn
}

ABSTRACT: A radiative natural SUSY spectrum are proposed in the deflected anomaly mediation scenario with general messenger-matter interactions. Due to the contributions from the new interactions, positive slepton masses as well as a large $\left|A_{t}\right|$ term can naturally be obtained with either sign of deflection parameter and few messenger species (thus avoid the possible Landau pole problem). In this scenario, in contrast to the ordinary (radiative) natural SUSY scenario with under-abundance of dark matter (DM), the DM can be the mixed bino-higgsino and have the right relic density. The $125 \mathrm{GeV}$ Higgs mass can also be easily obtained in our scenario. The majority of low EW fine tuning points can be covered by the XENON-1T direct detection experiments.

KeYwords: Supersymmetry Phenomenology

ARXIV EPrint: 1602.01699 


\section{Contents}

1 Introduction 1

2 A review on deflected AMSB with matter-messenger interactions 2

3 Deflected AMSB with new messenger-matter interactions 4

4 Radiative natural SUSY spectrum and numerical analysis $\quad 6$

$\begin{array}{llr}5 & \text { Conclusions } & 9\end{array}$

$\begin{array}{ll}\text { A Scalar soft SUSY breaking mass terms } & 10\end{array}$

\section{Introduction}

The standard model (SM) of particle physics has been confirmed by various experiments. Especially, a $125 \mathrm{GeV}$ SM-like Higgs boson was discovered by both the ATLAS [1] and CMS collaborations [2] of the Large Hadron Collider (LHC). On the other hand, the SM, as a successful effective theory, has many theoretical or aesthetical problems which necessitate various extensions. Low energy supersymmetry (SUSY) is a highly motivated paradigm for physics beyond the SM. In fact, an interesting observation is that the Higgs mass lies miraculously in the narrow $115-135 \mathrm{GeV}$ window predicted by the minimal SUSY model (MSSM). In addition, the top quark mass also lies exactly at what is needed to properly drive the radiative electroweak symmetry breaking (EWSB). Besides, the gauge hierarchy problem, the successful gauge coupling unification requirement as well as the dark matter (DM) puzzle can all be solved by SUSY.

The low energy SUSY paradigm is appealing, but so far there is no sign of SUSY particles after extensive searches at the LHC. In fact, no significant deviations from the SM have been observed in electroweak precision measurements as well as in flavor physics. The LHC data has already set stringent constraints [3-5] on certain CMSSM models: $m_{\tilde{g}} \gtrsim 1.8 \mathrm{TeV}$ for $m_{\tilde{q}} \sim m_{\tilde{g}}$, and $m_{\tilde{g}} \gtrsim 1.3 \mathrm{TeV}$ for $m_{\tilde{q}} \gg m_{\tilde{g}}$. Besides, the rather large value of the Higgs mass at $125 \mathrm{GeV}$ requires $\mathrm{TeV}$-scale highly mixed top squarks, which seems to contradict to the expectation from naturalness. In order to generate a soft SUSY spectrum that can be consistent with the LHC discoveries, a proper SUSY breaking mechanism is needed.

One of the most elegant SUSY breaking mechanisms is the anomaly mediation $[6,7]$ SUSY breaking scenario. The ordinary AMSB has many advantages and is very predictive. However, it has the tachyonic slepton problem [8-14] and needs an extension. An elegant extension to tackle the tachyonic slepton problem is the deflected AMSB scenario $[15,16]$, in which the messengers are introduced to deflect the Renormalization Group Equation 
(RGE) trajectory. The tachyonic slepton problem can be solved with such a deflection. On the other hand, many messenger species are needed to push slepton masses positive for a negative deflection parameter. A large number of messenger species could cause the Landau pole below the Planck scale. Besides, a large fine-tuning is needed to generate the $125 \mathrm{GeV}$ Higgs mass in the ordinary deflected AMSB scenario.

In our previous work [17], we proposed to introduce general messenger-matter interactions in the deflected AMSB scenario. The slepton sector can receive additional contributions from both the messenger-matter interactions and the ordinary deflected anomaly mediation to avoid tachyonic slepton masses. At the same time, additional contributions to trilinear coupling $A_{t}$ term which typically increase $\left|\tilde{A}_{t}\right|\left(\equiv A_{t}-\mu \cot \beta \mid\right)$ could be helpful to give the $125 \mathrm{GeV}$ Higgs and reduce the fine-tuning involved. Besides, even with one messenger we can generate positive slepton masses regardless the sign of deflection parameters [18-20]. So the Landau pole problem can be evaded in our new scenario.

Note that with a large $A_{t}$ term and the TeV-scale stops as well as a small $\mu \sim 100-$ $300 \mathrm{GeV}$, the radiative natural SUSY scenario [21-25] can naturally be realized in the deflected AMSB with general messenger-matter interactions. The electroweak (EW) finetuning [26-28] is small (typically $\Delta_{E W}<50$ ), especially when $A_{t}$ is large which will decrease the fine-tuning involved. On the other hand, the DM in ordinary natural SUSY will always be higgsino-like and results in under-abundance. Although two-component dark matter (axion and higgsino) can work well [29-33], it is preferable to change the nature of DM. We know that the gaugino mass relation in the ordinary AMSB is different from the relation in gauge mediation and gravity mediation. It will result in wino-higgsino DM and thus the under-abundance problem persists [34]. With the deflection of AMSB trajectory, the DM can be the mixed bino-higgsino and could give the right relic density. In this work we focus on such a realization of the radiative natural SUSY in the deflected AMSB with general messenger-matter interactions.

This paper is organized as follows. We briefly review the deflected AMSB scenario with general messenger-matter interactions in section 2 . In section 3 we introduce new messenger-matter interactions to the deflected AMSB and study the soft parameters which can generate the radiative natural SUSY. Numerical results are presented in section 4 . Section 5 contains our conclusions.

\section{A review on deflected AMSB with matter-messenger interactions}

We briefly review the general results of the deflected AMSB scenario with general mattermessenger interactions. The relevant details can be found in our previous study [17]. General messenger-matter interactions in GMSB can be seen in various papers [35-42].

The superpotential in the deflected AMSB scenario includes general messenger-matter interactions:

$$
W=\lambda_{\phi i j} X Q_{i} Q_{j}+y_{i j k} Q_{i} Q_{j} Q_{k}+W(X),
$$

where the indices ' $i, j^{\prime}$ run over all MSSM and messenger fields. Subscripts ' $U, D^{\prime}$ will denote the cases up and below the messenger threshold, respectively. $W(X)$ denotes the superpotential for pseduo-moduli field $X$ which defines the messenger threshold. 
After integrating out the messenger fields, we have the general form for the MSSM fields only:

$$
\mathcal{L}=\int d^{4} \theta Q_{a}^{\dagger} Z_{D}^{a b}\left(\frac{\mu}{\sqrt{\phi^{\dagger} \phi}}, \sqrt{\frac{X^{\dagger} X}{\phi^{\dagger} \phi}}\right) Q_{b}+\int d^{2} \theta y_{a b c} Q^{a} Q^{b} Q^{c},
$$

which can give additional contributions to soft SUSY breaking parameters. Here ' $\phi$ ' denotes the compensator field with Weyl weight 1 and ' $Z_{D}^{\prime}$ the wavefunction renormalization factor below the messenger threshold.

The leading-order contributions to the trilinear terms and scalar terms are

$$
\begin{aligned}
\frac{A_{a b c}}{y_{a b c}} & =\sum_{i=a, b, c}\left(-\frac{1}{2} F_{\phi} \frac{\partial}{\partial \ln \mu}+\frac{d F_{\phi}}{2} \frac{\partial}{\partial \ln |\tilde{X}|}\right) \ln Z_{D}^{i i}(\mu,|\tilde{X}|), \\
m_{a b}^{2} & =\left(-\frac{\left|F_{\phi}\right|^{2}}{4} \frac{\partial^{2}}{\partial(\ln \mu)^{2}}-\frac{\left|F_{\tilde{X}}\right|^{2}}{4} \frac{\partial^{2}}{\partial|\tilde{X}|^{2}}+\frac{\left|F_{\phi}\right|\left|F_{\tilde{X}}\right|}{2} \frac{\partial^{2}}{\partial \ln \mu \partial|\tilde{X}|}\right) \ln Z_{D}^{a b}(\mu,|\tilde{X}|), \\
& =\left[-\frac{\left|F_{\phi}\right|^{2}}{4} \frac{\partial^{2}}{\partial(\ln \mu)^{2}}-\frac{d^{2}\left|F_{\phi}\right|^{2}}{4} \frac{\partial^{2}}{\partial \ln |\tilde{X}|^{2}}+\frac{d\left|F_{\phi}\right|^{2}}{2} \frac{\partial^{2}}{\partial \ln \mu \partial \ln |\tilde{X}|}\right] \ln Z_{D}^{a b}(\mu,|\tilde{X}|),
\end{aligned}
$$

where the last term is the unique feature of this deflected AMSB scenario which involves the interference between the pure anomaly and gauge mediation type contributions.

Following the conventions in [36], the derivative of the wavefunction with respect to $t=\ln \mu$ are given as

$$
\begin{aligned}
\frac{d Z_{i j}}{d t} & \equiv G_{i j}[Z(\ln \mu) ; \lambda(\ln \mu) ; g(\ln \mu)], \\
& =-\frac{1}{8 \pi^{2}}\left(\frac{1}{2} d_{i}^{k l} \lambda_{i k l}^{*} Z_{k m}^{-1}{ }^{*} Z_{l n}^{-1}{ }^{*} \lambda_{j m n}-2 c_{r}^{i} Z_{i j} g_{r}^{2}\right),
\end{aligned}
$$

we can obtain the expression for the first derivative of wavefunction with respect to ' $X^{\prime}$ [35] at the messenger scale $\mu=|X|$

$$
\frac{\partial Z_{D}^{a b}(\ln \mu,|X|)}{\partial X}=\frac{1}{2 X} \Delta G^{a b}
$$

with $\Delta(\cdots)$ denoting the discontinuity of its followed expression, and $d_{i}^{k l}$ being the standard multiplicity factor in the one-loop anomalous dimensions.

The interference terms between the anomaly mediation and gauge mediation are

$$
\begin{aligned}
& \frac{\partial^{2}}{\partial \ln \mu \partial \ln |\tilde{X}|} Z_{D}^{a}(\mu,|\tilde{X}|)=\frac{\partial}{\partial \ln |\tilde{X}|} G_{a}\left[Z_{D}(\ln \mu, \tilde{X}) ; \lambda(\ln \mu, \tilde{X}) ; g(\ln \mu, \tilde{X})\right], \\
& =\left(\Delta\left(\beta_{\lambda}\right) \frac{\partial}{\partial \lambda}+\Delta\left(\beta_{g}\right) \frac{\partial}{\partial g}+\frac{\partial Z_{D}^{a}}{\partial \ln \tilde{X}} \frac{\partial}{\partial Z_{D}^{a}}\right) G_{a}\left[Z_{D}^{a}(\ln \mu) ; \lambda(\ln \mu) ; g(\ln \mu)\right] .
\end{aligned}
$$

So we arrive at the final results for the trilinear and scalar soft masses with a general messenger sector at the messenger scale [17]:

$$
A_{a}=-\frac{1}{2} G_{a a}^{D} F_{\phi}-\frac{1}{32 \pi^{2}} d_{a}^{i j} \Delta\left(\left|\lambda_{a i j}\right|^{2}\right) d F_{\phi}
$$




$$
m^{2}=m_{\mathrm{AMSB}}^{2}+m_{\text {gauge }}^{2}+m_{\mathrm{inter}}^{2},
$$

with

$$
\begin{aligned}
m_{\mathrm{AMSB}}^{2}= & {\left[-\frac{\left|F_{\phi}\right|^{2}}{4}\left(\frac{\partial \gamma^{a}}{\partial g_{i}} \beta\left(g_{i}\right)+\frac{\partial \gamma^{a}}{\partial y_{i}} \beta\left(y_{i}\right)\right)\right] } \\
\left(m_{a b}^{2}\right)_{\text {inter }}= & \frac{d F_{\phi}^{2}}{2}\left\{-\frac{1}{8 \pi^{2}}\left[d_{a}^{k l} \lambda_{a k l}^{*} \lambda_{b m n}\left(\frac{\Delta G_{k m}^{D}}{2}+\frac{\Delta G_{l n}^{D}}{2}\right)+2 c_{r}^{i} g_{r}^{2} \frac{\Delta G_{a b}^{D}}{2}\right]\right. \\
& \left.+\frac{1}{8 \pi^{2}} 4 c_{r}^{k} \frac{1}{16 \pi^{2}} g_{k}^{4} \Delta\left(b_{k}\right)-G_{D} \frac{\Delta G_{D}}{2}\right\}
\end{aligned}
$$

and the gauge mediation type contributions similar to [36]:

$$
\begin{aligned}
\left(m_{a b}^{2}\right)_{\text {gauge }}= & \frac{d^{2} F_{\phi}^{2}}{4} \frac{1}{256 \pi^{4}}\left[\frac{1}{2} d_{a}^{i k} d_{i}^{l m}\left(\Delta\left(\lambda_{a i k}^{*} \lambda_{b j k}\right)\left(\lambda_{i l m} \lambda_{j l m}^{*}\right)^{U}\right)-\left(\lambda_{a i k}^{*} \lambda_{b j k}\right)^{D} \Delta\left(\lambda_{i l m} \lambda_{j l m}^{*}\right)\right. \\
& \left.+\frac{1}{4} d_{a}^{i j} d_{b}^{k l} \Delta\left(\lambda_{a i j}^{*} \lambda_{c i j}\right) \Delta\left(\lambda_{c k l}^{*} \lambda_{b k l}\right)-d_{a}^{i j} C_{r}^{a i j} g_{r}^{2} \Delta\left(\lambda_{a i j}^{*} \lambda_{b i j}\right)\right]
\end{aligned}
$$

\section{Deflected AMSB with new messenger-matter interactions}

The characteristic feature of the radiative natural SUSY with respect to the ordinary natural SUSY is the large $\left|A_{t}\right|$ term. In order to obtain the relatively large trilinear terms, we include new messenger-matter interactions in the deflected AMSB scenario. The messengers are introduced in pairs of $(\mathbf{5}, \overline{\mathbf{5}})$ representations of $\mathrm{SU}(5)$. So the messengers obviously have the following decomposition in terms of the $\mathrm{SM} \mathrm{SU}(3)_{c} \times \mathrm{SU}(2)_{L} \times \mathrm{U}(1)_{Y}$ quantum number:

$$
\begin{aligned}
\mathbf{5}_{a} & =N_{a}(1,2)_{1 / 2} \oplus M_{a}(3,1)_{-1 / 3}, \\
\overline{\mathbf{5}}_{a} & =\bar{N}_{a}(1, \overline{2})_{-1 / 2} \oplus \bar{M}_{a}(\overline{3}, 1)_{1 / 3},
\end{aligned}
$$

with ' $a$ ' denoting the $N_{F}$ messenger species.

We introduce the following superpotential that involves the messenger-MSSM interaction

$$
\begin{aligned}
W^{U} \supset & X \bar{N}_{a} N_{a}+X \bar{M}_{a} M_{a}+W(X) \\
& +\sum_{i}\left[\lambda_{a i}^{D} Q_{L}^{i}\left(D_{R}^{c}\right)_{i} \bar{N}_{a}+\lambda_{a i}^{L} L_{i}\left(E_{L}^{c}\right)_{i} \bar{N}_{a}+\lambda_{a i}^{U} Q_{L}^{i}\left(U_{R}^{c}\right)_{i} N_{a}\right],
\end{aligned}
$$

with the typical form of superpotential $W(X)$ for pseduo-moduli field $X$ to determine the deflection parameter $d$ in combination with $F_{\phi}$. Here the superscript ${ }^{\prime} i{ }^{\prime}$ denotes the family indices.

From the general expressions of soft parameters in section 2, we can obtain the soft SUSY breaking parameters for sfermions and trilinear couplings at the messenger scale. We keep the leading terms involving only $y_{t}, g_{3}, \lambda_{L, i}, \lambda_{U, i}, \lambda_{D, i}$. Subleading terms like $g_{1,2}^{4}, y_{b, \tau}^{2} \lambda_{L, U, D ; i a}^{2}$ are not kept in the following expressions. For simplicity, we set family and messenger species universal couplings $\lambda_{L, a i}=\lambda_{L} ; \lambda_{U, a i}=\lambda_{U} ; \lambda_{D, a i}=\lambda_{D}$ for messengermatter interactions. Besides, we only give explicitly the soft terms for the third generation 
squarks. The first two generation squarks can be obtained by removing the $y_{t}^{2}$ terms in the relevant expressions. The soft SUSY mass terms for the three generations of sleptons have the same form. The values of $\mu$ and $B \mu$ are model-dependent and we leave them as free parameters because we do not give an explicit mechanism in our scenario. They are determined by successful EWSB conditions.

The gaugino masses are given by

$$
M_{i}=-\frac{\alpha_{i}}{4 \pi}\left(b_{i}+N_{F} d\right)
$$

with the beta function $\left(b_{1}, b_{2}, b_{3}\right)=(33 / 5,1,-3)$ and the standard normalization for $g_{1}$ coupling $g_{1}^{2}=5 g_{Y}^{2} / 3$.

The trilinear couplings are calculated to be

$$
\begin{aligned}
& A_{t}=\frac{F_{\phi}}{16 \pi^{2}}\left[6 y_{t}^{2}-\left(3 \lambda_{U}^{2}+\lambda_{D}^{2}\right) d-\frac{16}{3} g_{3}^{2}\right] \\
& A_{b}=\frac{F_{\phi}}{16 \pi^{2}}\left[y_{t}^{2}-\left(\lambda_{U}^{2}+3 \lambda_{D}^{2}\right) d-\frac{16}{3} g_{3}^{2}\right] \\
& A_{\tau}=\frac{F_{\phi}}{16 \pi^{2}}\left(-3 \lambda_{L}^{2} d\right)
\end{aligned}
$$

The soft parameters are

$$
\frac{m^{2}}{F_{\phi}^{2}}=\frac{d}{2} \delta^{m}+\frac{d^{2}}{4}\left(\delta^{G}+\delta^{3}\right)+\frac{1}{4} \delta^{A}
$$

with the relevant tedious expressions given in the appendix.

We have the following discussions:

(i) In our scenario, the notorious tachyonic slepton problem which appears in the ordinary AMSB can be naturally solved. Besides, the slepton masses receive (dominant) positive contributions from matter-messenger interactions regardless of the sign of the deflection parameter $d$.

(ii) In our scenario, even one messenger specie can work well to give positive slepton masses regardless of the sign of deflection parameter $d$. So the possible Landau pole problem below the Planck scale will naturally be evaded in our scenario.

(iii) The $A_{t}$ value can be either positive or negative, depending on the sign of $d$. Large $\lambda_{U, D}$ can lead to a large value of $\left|A_{t}\right|$ which can naturally give a large Higgs mass with a less fine-tuning.

(iv) There is some parameter space for light soft stop masses. So the radiative natural SUSY spectrum can be realized in our scenario. We will discuss such a realization in next section. 


\section{Radiative natural SUSY spectrum and numerical analysis}

The $125 \mathrm{GeV}$ Higgs has already set some constraints on the low energy SUSY spectrum. Obviously from the formula

$$
m_{h}^{2} \simeq m_{Z}^{2} \cos ^{2} 2 \beta+\frac{3 m_{t}^{4}}{4 \pi^{2} v^{2}}\left[\log \frac{M_{\text {SUSY }}^{2}}{m_{t}^{2}}+\frac{\tilde{A}_{t}^{2}}{M_{\text {SUSY }}^{2}}\left(1-\frac{\tilde{A}_{t}^{2}}{12 M_{\text {SUSY }}^{2}}\right)\right],
$$

with

$$
\tilde{A}_{t}=A_{t}-\mu \cot \beta, \quad M_{\mathrm{SUSY}}^{2}=m_{\tilde{t}_{1}} m_{\tilde{t}_{2}},
$$

we need either $M_{\mathrm{SUSY}} / m_{t} \gg 1$ or $M_{\mathrm{SUSY}} / m_{t}>1$ with $\tilde{A}_{t} / M_{\mathrm{SUSY}}>1$. The stop masses must be larger than $10 \mathrm{TeV}$ in case of no stop mixing, and hence a large fine tuning is needed. Obviously, a large $\tilde{A}_{t}$ is preferable for low energy SUSY.

The models of natural SUSY [21-24] try to retain the naturalness of weak scale SUSY by proposing a spectrum of light higgsinos $|\mu| \sim 100-300 \mathrm{GeV}$ and light $\tilde{t}_{1,2}, \tilde{b}_{1}$ along with very heavy masses of other squarks and $\mathrm{TeV}$-scale gluinos. The gluino mass can affect the stop masses via RGE evolution. So, a low EW fine-tuning requires that the gluino mass can not be too heavy. On the other hand, it is also bounded from below to be $m_{\tilde{g}} \gtrsim 1.3 \mathrm{TeV}$ by the LHC searches within the context of SUSY models like mSUGRA/CMSSM. The first two generation sfermions can be allowed to lie in the $5-20 \mathrm{TeV}$ range without introducing unnaturalness. Heavier first two generation squarks can ameliorates the SUSY flavour, $\mathrm{CP}$, gravitino and proton-decay problems due to decoupling. Such models have a low electroweak fine-tuning and satisfy the LHC constraints.

However, the relatively heavy $(125 \mathrm{GeV})$ Higgs mass has some tension with the ordinary natural SUSY scenario and indicates that natural SUSY may take the form of radiative natural SUSY [25] which requires a large $A_{t}$ term. In fact, a large $\left|A_{t}\right|$ value can suppress the top squark contributions to $\Sigma_{u}^{u}$ and at the same time lift up the Higgs mass. Such a large $\left|A_{t}\right|$ can easily be obtained in our scenario. We can see from eq. (3.3) that a large $\left|A_{t}\right|$ will appear in case of a large $\lambda$ and either sign of deflection parameter $d$.

In the ordinary radiative natural SUSY scenario with universal gaugino mass at the GUT scale, the lightest sparticle (LSP) is always the higgsino which can not fully account for the DM relic abundance. The gaugino relation at the EW scale can naturally be evaded in the deflected AMSB scenario and thus the DM can be the mixed bino-higgsino or winohiggsino (or pure bino, pure wino). We know that in the ordinary AMSB, the gaugino mass ratio at the EW scale is

$$
M_{1}: M_{2}: M_{3} \approx 3.29: 1:-9.6 .
$$

This can lead to the mixed higgsino-wino dark matter for gluino at about $2 \mathrm{TeV}$. As noted in [34], the under-abundance problem of DM persists. In general, in order to get the mixed higgsino-electroweakino DM, we need the gaugino mass ratio to satisfy

$$
M_{3}: \min \left(M_{1}, M_{2}\right) \gtrsim 5,
$$

with gluino mass heavier than $1.5 \mathrm{TeV}$. The mixed bino-higgsino DM can give the full DM abundance. This prefer a negative deflection parameter with $N_{F} d \lesssim-3$. 
In our scenario, the soft terms are characterized by the following free parameters

$$
N_{F}, d, \mu, M_{\text {mess }}, F_{\phi}, \tan \beta, \lambda_{U}, \lambda_{D}, \lambda_{L}
$$

We scan the parameter space with the following messenger scale $\left(M_{\text {mess }}\right)$ inputs:

- The $\mu$ parameter is chosen to lie between $|\mu| \sim 100-300 \mathrm{GeV}$ to keep EW naturalness.

- The scale of $F_{\phi}$ determines the whole SUSY spectrum. The gaugino masses, the EWSB condition as well the Higgs mass constrain the value of $F_{\phi}$ to be in the range $10 \mathrm{TeV}<F_{\phi}<500 \mathrm{TeV}$.

- The messenger scale $M_{\text {mess }}$ can be chosen to lie between the GUT scale and the typical sparticle scale: $10 \mathrm{TeV}<M_{\text {mess }}<10^{16} \mathrm{GeV}$.

- The value of $\tan \beta$ is chosen to be $40 \geq \tan \beta \geq 2$. The messenger species $N_{F}$ should lie in the range $1 \leq N_{F} \leq 3$ to avoid the possible Landau pole while the deflection parameter $d$ is chosen to satisfy $N_{F} \cdot d \lesssim-3$ to fully account for the DM relic density.

- For simplicity, we set $\lambda_{U}=\lambda_{D}=\lambda$. We set the range of the messenger-matter interactions: $0.5 \lesssim \lambda, \lambda_{L} \lesssim 3$ to justify our keeping of the leading contributions in previous calculations and at the same time avoid the possible Landau pole.

In our scan we take into account the following collider and dark matter constraints:

(1) Successful radiative EWSB condition.

(2) The stop and sbottom masses can be relatively heavy in the radiative natural SUSY scenario in contrast to the upper bound of $1.5 \mathrm{TeV}$ in ordinary natural SUSY (with less than $10 \% \mathrm{EW}$ fine tuning ). We require that the stop masses to satisfy $m_{\tilde{t}_{1,2}} \lesssim$ $4 \mathrm{TeV}$ which corresponds to to an upper bound for the EW fine-tuning $\Delta_{E W} \lesssim 50$. A large $\left|A_{t}\right|$ will always decrease the fine-tuning involved. Due to the gluino loop contribution to the stop masses, the gluino is bounded to be below $12 \mathrm{TeV}$.

(3) The lower bounds on neutralino and chargino masses from LEP, including the invisible decay of $Z$-boson. The most stringent constraints of LEP come from the chargino mass and the invisible $Z$-boson decay. We require $m_{\tilde{\chi}^{ \pm}}>103.5 \mathrm{GeV}$ and the invisible decay width $\Gamma\left(Z \rightarrow \tilde{\chi}_{0} \tilde{\chi}_{0}\right)<1.71 \mathrm{MeV}$, which is consistent with the $2 \sigma$ precision EW measurement $\Gamma_{\text {inv }}^{\text {non-SM }}<2.0 \mathrm{MeV}$.

(4) The combined mass range for the Higgs boson: $123 \mathrm{GeV}<M_{h}<127 \mathrm{GeV}$ from ATLAS and CMS data $[1,2]$.

(5) The relic density of the neutralino dark matter satisfies the Planck result $\Omega_{D M}=$ $0.1199 \pm 0.0027$ [43] (in combination with the WMAP data [44]) with a $10 \%$ theoretical uncertainty). 

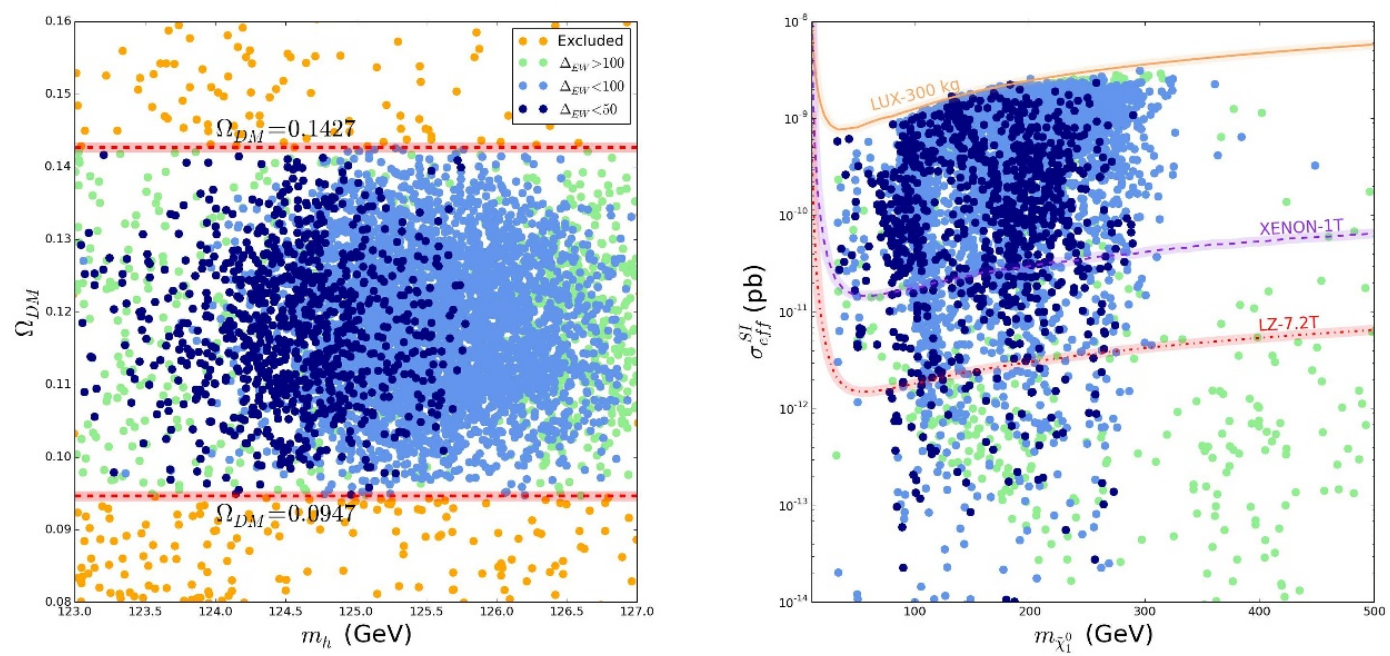

Figure 1. The scatter plots of the parameter space in our scenario, showing the dark matter relic density versus the Higgs mass in the left panel and the spin-independent DM-nucleon scattering cross section versus the LSP mass in the right panel. All the points can survive the collider and dark matter constraints (1-6). The EW fine tuning $\left(\Delta_{E W}\right)$ for the sample points are also shown.

(6) The dark matter in our scenario can be the mixed bino-higgsino. In this case, the direct detection experiments can possibly set stringent constraints on dark matter. We survey the spin-independent (SI) direct detection bounds from LUX [45], Xeon1T [46] and the future LUX-ZEPLIN 7.2 Ton [47] experiment.

The numerical results with the corresponding EW fine-tuning are shown in Fig 1. It should be noted that [49] conventional measures, include BG measure [48], tend to overestimate EWFT in supersymmetric models, often by several orders of magnitude. Accord to the Fine-tuning Rule proposed in [50], both Higgs mass and the traditional $\Delta_{B G}$ fine-tuning measures reduce to the model-independent EW fine-tuning measure $\Delta_{E W}$.

From the figure, we have the following observations:

- Both the $125 \mathrm{GeV}$ Higgs mass and the correct DM relic density can be obtained in our scenario. We can see that there is a large parameter space which can give the correct relic abundance of DM. This is the consequence of the mixed bino-higgsino DM nature in our scenario. The deflection of AMSB trajectory is crucial for a light bino to be the lightest gaugino (with $M_{1} \lesssim \mu$ ) that can be compatible with the LHC constraints on gluino mass $m_{\tilde{g}} \gtrsim 1.3 \mathrm{TeV}$. Without the deflection, the lightest gaugino would be heavy and at the same time wino-like. Such a relation would predict either higgsino or mixed wino-higgsino DM, both of which would lead to under-abundance of DM.

- Our scenario can also give the observed $125 \mathrm{GeV}$ Higgs mass. This is the consequence of a relatively large $A_{t}$ term. Besides, the EW fine-tuning needed for the $125 \mathrm{GeV}$ Higgs mass can be as low as $\Delta_{E W} \lesssim 50$. Larger higgs mass will slight increase the EW fine tuning involved. 
- We also survey the spin-independent (SI) direct detection bounds from DM-nucleon scattering experiments. It is well known that the SI interaction of the neutralino DM with quarks inside the nucleus occurs via the $s$-channel squark exchange and $t$-channel Higgs exchange processes. As squarks are bounded by the LHC data to be considerably heavy, the Higgs exchange diagrams would dominantly contribute to the spin-independent $\chi-p$ scattering cross section. The Higgs- $\chi-\chi$ coupling is driven by bino-higgsino and wino-higgsino mixing. Unlike the case for a pure gaugino or a pure higgsino DM in which the associated SI cross-section would become quite small, the SI cross section could be large when DM is the mixed bino-higgsino. However, the DM can evade the SI direct detection experiments if the mixing is small. In our numerical study, we find that the most interesting points with low EW fine tuning(namely the points that can account for the $125 \mathrm{GeV}$ Higgs mass with $\Delta_{E W}<100$ ) have typically a cross section below $10^{-9} \mathrm{pb}$. The majority of such points will be covered by XENON-1T. On the other hand, there are still small regions with low EW fine tuning that can survive the XENON-1T and LUX-ZEPLIN 7.2 Ton sensitivity. Such points may indicates that the corresponding mixing of bino-higgsino is not large.

\section{Conclusions}

In this work a radiative natural SUSY spectrum were proposed in deflected anomaly mediation scenario with general messenger-matter interactions. Due to the contributions from new interactions, positive slepton masses as well as a large $\left|A_{t}\right|$ term can naturally be obtained with either sign of deflection parameter and few messenger species (thus avoid the possible Landau pole problem). In this scenario, in contrast to the ordinary radiative natural SUSY scenario with under-abundance of DM, the DM can be the mixed bino-higgsino and give the right relic density. The $125 \mathrm{GeV}$ Higgs mass can also be easily obtained in our scenario. The majority of low EW fine tuning points can be covered by the XENON-1T direct detection experiments.

\section{Acknowledgments}

We are very grateful to the referee for helpful discussions. This work was supported by the National Natural Science Foundation of China (NNSFC) under grant No. 11105124, 11105125, 11135003 and 11275245, by the Open Project Program of State Key Laboratory of Theoretical Physics, Institute of Theoretical Physics, Chinese Academy of Sciences (No.Y5KF121CJ1), by the Innovation Talent project of Henan Province under grant number 15HASTIT017, by the Young-Talent Foundation of Zhengzhou University, and by the CAS Center for Excellence in Particle Physics (CCEPP). 


\section{A Scalar soft SUSY breaking mass terms}

The expression for the scalar soft parameters are derived from the general forms in [17] and given by

$$
\frac{m^{2}}{F_{\phi}^{2}}=\frac{d}{2} \delta^{m}+\frac{d^{2}}{4}\left(\delta^{G}+\delta^{3}\right)+\frac{1}{4} \delta^{A}
$$

with each type of contributions given below. The relevant expressions are

- Cross term (anomaly-gauge mediation) contributions:

the anomaly-gauge mixed mediation part given by

$$
\begin{aligned}
\delta^{m} & =\frac{\partial^{2}}{\partial \mu \partial \ln |X|} \ln Z_{a b}^{D}, \\
& =\left(\frac{\Delta G_{a}^{D}}{2} \frac{\partial}{\partial Z_{a}^{D}}+\Delta \beta_{g_{r}} \frac{\partial}{\partial g_{r}}\right) G^{-}-G_{a}^{D} \frac{\Delta G_{a}}{2} .
\end{aligned}
$$

Cross the messenger threshold, the change of the beta function for $g_{i}$ is given by

$$
\Delta \beta_{g_{i}}=\frac{1}{16 \pi^{2}} N_{F} g_{i}^{3}
$$

and the discontinuity of $G^{a}$ is

$$
\begin{aligned}
\frac{\Delta G_{L}}{2} & =-\frac{1}{8 \pi^{2}} \lambda_{L}^{2}, \\
\frac{\Delta G_{E_{L}^{c}}}{2} & =-\frac{1}{8 \pi^{2}} 2 \lambda_{L}^{2}, \\
\frac{\Delta G_{Q_{L}}}{2} & =-\frac{1}{8 \pi^{2}}\left(\lambda_{D}^{2}+\lambda_{U}^{2}\right), \\
\frac{\Delta G_{U_{L}^{c}}}{2} & =-\frac{1}{8 \pi^{2}}\left(2 \lambda_{U}^{2}\right), \\
\frac{\Delta G_{D_{L}^{c}}}{2} & =-\frac{1}{8 \pi^{2}}\left(2 \lambda_{D}^{2}\right) .
\end{aligned}
$$

After some manipulations, we can obtain

$$
\begin{aligned}
\delta_{Q}^{m} & =\frac{1}{8 \pi^{2}}\left[y_{t}^{2} \frac{\Delta G_{y_{t}}}{2}+y_{b}^{2} \frac{\Delta G y_{b}}{2}\right]+\Delta \beta_{g_{r}} \frac{\partial}{\partial g_{r}} G_{Q}^{D}, \\
& \approx-\frac{1}{8 \pi^{2}}\left[2 y_{t}^{2} \frac{1}{16 \pi^{2}}\left(3 \lambda_{U}^{2}+\lambda_{D}^{2}\right)-2 \frac{8}{3} \frac{1}{16 \pi^{2}} N_{F} g_{3}^{4}\right], \\
\delta_{U}^{m} & =-\frac{1}{8 \pi^{2}}\left[4 y_{t}^{2} \frac{1}{16 \pi^{2}}\left(3 \lambda_{U}^{2}+\lambda_{D}^{2}\right)-2 \frac{8}{3} \frac{1}{16 \pi^{2}} N_{F} g_{3}^{4}\right], \\
\delta_{D}^{m} & =-\frac{1}{8 \pi^{2}}\left[-2 \frac{8}{3} \frac{1}{16 \pi^{2}} N_{F} g_{3}^{4}\right] \\
\delta_{L}^{m} & =\delta_{E}^{m}=\delta_{H_{D}}^{m}=0 \\
\delta_{H_{U}}^{m} & =-\frac{1}{8 \pi^{2}}\left[6 y_{t}^{2} \frac{1}{16 \pi^{2}}\left(3 \lambda_{U}^{2}+\lambda_{D}^{2}\right)\right],
\end{aligned}
$$

Expressions for the first two generation squarks can be obtained by simply removing the $y_{t}^{2}$ terms. 
- Gauge mediation-type contributions:

the gauge mediation part given by

$$
\delta^{G}+\delta^{3}=-\frac{\partial^{2}}{\partial \ln |X|^{2}} \ln Z=-\frac{\partial^{2}}{\partial \ln |X|^{2}} Z+\left|\frac{\partial Z}{\partial \ln |X|}\right|^{2} .
$$

The sums of the discontinuity are

$$
\begin{aligned}
& \sum \Delta\left(\frac{\partial G^{Q}}{\partial Z_{a}}\right) G_{a}=\frac{1}{8 \pi^{2}}\left[\lambda_{U}^{2}\left(G_{\lambda_{U}}-G_{Q}\right)+\lambda_{D}^{2}\left(G_{\lambda_{D}}-G_{Q}\right)\right] \\
& \sum \Delta\left(\frac{\partial G^{U}}{\partial Z_{a}}\right) G_{a}=\frac{1}{8 \pi^{2}}\left[2 \lambda_{U}^{2}\left(G_{\lambda_{U}}-G_{U}\right)\right] \\
& \sum \Delta\left(\frac{\partial G^{D}}{\partial Z_{a}}\right) G_{a}=\frac{1}{8 \pi^{2}}\left[2 \lambda_{D}^{2}\left(G_{\lambda_{D}}-G_{D}\right)\right] \\
& \sum \Delta\left(\frac{\partial G^{L}}{\partial Z_{a}}\right) G_{a}=\frac{1}{8 \pi^{2}}\left[\lambda_{L}^{2}\left(G_{\lambda_{L}}-G_{L}\right)\right] \\
& \sum \Delta\left(\frac{\partial G^{E}}{\partial Z_{a}}\right) G_{a}=\frac{1}{8 \pi^{2}}\left[\lambda_{U}^{2}\left(G_{\lambda_{L}}-G_{E}\right)\right]
\end{aligned}
$$

with $G_{\lambda_{U}}^{U}=G_{Q}^{U}+G_{U}^{U}+G_{X_{u}}^{U}$ and $G_{\lambda_{D}}^{U}=G_{Q}^{U}+G_{D}^{U}+G_{X_{d}}^{U}$ the anomalous dimension for $\lambda_{U}$ and $\lambda_{D}$ above the threshold. So we can obtain

$$
\begin{aligned}
\delta_{Q}^{G} & =\frac{1}{8 \pi^{2}}\left[y_{t}^{2} \frac{\Delta G_{y_{t}}}{2}-\lambda_{U}^{2} G_{\lambda_{U}}^{T U}-\lambda_{D}^{2} G_{\lambda_{D}}^{T U}\right], \\
\delta_{U}^{G} & =\frac{1}{8 \pi^{2}}\left[2 y_{t}^{2} \frac{\Delta G_{y_{t}}}{2}-2 \lambda_{U}^{2} G_{\lambda_{U}}^{T U}\right], \\
\delta_{D}^{G} & =\frac{1}{8 \pi^{2}}\left[-2 \lambda_{D}^{2} G_{\lambda_{D}}^{T U}\right], \\
\delta_{L}^{G} & =\frac{1}{8 \pi^{2}}\left[-\lambda_{L}^{2} G_{\lambda_{L}}^{T U}\right], \\
\delta_{E}^{G} & =\frac{1}{8 \pi^{2}}\left[-2 \lambda_{L}^{2} G_{\lambda_{L}}^{T U}\right], \\
\delta_{H_{D}}^{G} & =0 \\
\delta_{H_{U}}^{G} & =\frac{1}{8 \pi^{2}}\left[3 y_{t}^{2} \frac{\Delta G_{y_{t}}}{2}-3 \lambda_{U}^{2} G_{\lambda_{U}}^{T U}\right],
\end{aligned}
$$

The index TU denotes the value upon the messenger threshold. We list their expressions:

$$
\begin{aligned}
\frac{\Delta G_{y_{t}}}{2} & =-\frac{1}{8 \pi^{2}}\left(3 \lambda_{U}^{2}+\lambda_{D}^{2}\right), \\
\frac{\Delta G_{y_{b}}}{2} & =-\frac{1}{8 \pi^{2}}\left(\lambda_{U}^{2}+3 \lambda_{D}^{2}\right), \\
G_{\lambda_{U}}^{T U} & =-\frac{1}{8 \pi^{2}}\left(6 \lambda_{U}^{2}+\lambda_{D}^{2}+3 y_{t}^{2}-\frac{16}{3} g_{3}^{2}\right), \\
G_{\lambda_{D}}^{T U} & =-\frac{1}{8 \pi^{2}}\left(6 \lambda_{D}^{2}+\lambda_{U}^{2}+\lambda_{L}^{2}+y_{t}^{2}-\frac{16}{3} g_{3}^{2}\right), \\
G_{\lambda_{L}}^{T U} & =-\frac{1}{8 \pi^{2}}\left(4 \lambda_{L}^{2}+3 \lambda_{D}^{2}\right) .
\end{aligned}
$$


There are other terms from ordinary GMSB part with

$$
\delta^{3}=\Delta \beta_{g_{r}}\left(\frac{\partial}{\partial g_{r}} G^{T D}\right)=\frac{1}{8 \pi^{2}} 2 c_{r} 2 g_{r} \frac{N_{F}}{16 \pi^{2}} g_{r}^{3} .
$$

Note that the change of the beta function is $\Delta \beta_{g}=N_{F}$.

$$
\begin{aligned}
& \delta_{Q}^{3}=\delta_{U}^{3}=\delta_{D}^{3}=\frac{N_{F}}{\left(8 \pi^{2}\right)^{2}}\left[\frac{8}{3} g_{3}^{4}\right], \\
& \delta_{L}^{3}=\delta_{E}^{3}=\delta_{H_{D}}^{3}=\delta_{H_{U}}^{3} \approx 0 .
\end{aligned}
$$

In the previous expressions, we keep the terms involving only $g_{3}$.

- Pure anomaly contributions:

$$
\delta^{A}=-\frac{\partial^{2}}{\partial \ln |X|^{2}} \ln Z=-\frac{\partial^{2}}{\partial \ln |X|^{2}} Z+\left|\frac{\partial Z}{\partial \ln |X|}\right|^{2} .
$$

So we obtain

$$
\begin{aligned}
\delta_{Q}^{A} & =-\frac{1}{8 \pi^{2}}\left[y_{t}^{2} G_{y_{t}}+y_{b}^{2} G_{y_{b}}\right]-\frac{1}{4 \pi^{2}}\left[\frac{1}{30} b_{1} \alpha_{1}^{2}+\frac{3}{2} b_{2} \alpha_{2}^{2}+\frac{8}{3} b_{3} \alpha_{3}^{2}\right], \\
& \approx \frac{1}{\left(8 \pi^{2}\right)^{2}}\left[y_{t}^{2}\left(6 y_{t}^{2}-\frac{16}{3} g_{3}^{2}\right)\right]-\frac{1}{4 \pi^{2}} \frac{8}{3} b_{3} \alpha_{3}^{2}, \\
\delta_{U}^{A} & =-\frac{1}{8 \pi^{2}}\left[2 y_{t}^{2} G_{y_{t}}\right]-\frac{1}{4 \pi^{2}}\left[\frac{8}{15} b_{1} \alpha_{1}^{2}+\frac{8}{3} b_{3} \alpha_{3}^{2}\right], \\
& \approx \frac{1}{\left(8 \pi^{2}\right)^{2}}\left[2 y_{t}^{2}\left(6 y_{t}^{2}-\frac{16}{3} g_{3}^{2}\right)\right]-\frac{1}{4 \pi^{2}} \frac{8}{3} b_{3} \alpha_{3}^{2}, \\
\delta_{D}^{A} & \approx-\frac{1}{4 \pi^{2}} \frac{8}{3} b_{3} \alpha_{3}^{2}, \\
\delta_{H_{u}}^{A} & =\frac{1}{\left(8 \pi^{2}\right)^{2}} 3 y_{t}^{2}\left(6 y_{t}^{2}-\frac{16}{3} g_{3}^{2}\right), \\
\delta_{L}^{A} & =\delta_{E}^{A}=\delta_{H_{d}}^{A} \approx 0 .
\end{aligned}
$$

Open Access. This article is distributed under the terms of the Creative Commons Attribution License (CC-BY 4.0), which permits any use, distribution and reproduction in any medium, provided the original author(s) and source are credited.

\section{References}

[1] ATLAS collaboration, Combined search for the standard model Higgs boson using up to $4.9 \mathrm{fb}^{-1}$ of pp collision data at $\sqrt{s}=7 \mathrm{TeV}$ with the ATLAS detector at the LHC,

Phys. Lett. B 710 (2012) 49 [arXiv:1202.1408] [INSPIRE].

[2] CMS collaboration, Combined results of searches for the standard model Higgs boson in pp collisions at $\sqrt{s}=7$ TeV, Phys. Lett. B 710 (2012) 26 [arXiv:1202.1488] [INSPIRE].

[3] ATLAS collaboration, Search for squarks and gluinos using final states with jets and missing transverse momentum with the ATLAS detector in $\sqrt{s}=7$ TeV proton-proton collisions, Phys. Lett. B 710 (2012) 67 [arXiv:1109.6572] [INSPIRE]. 
[4] ATLAS collaboration, Search for squarks and gluinos with the ATLAS detector in final states with jets and missing transverse momentum using $4.7 \mathrm{fb}^{-1}$ of $\sqrt{s}=7 \mathrm{TeV}$ proton-proton collision data, Phys. Rev. D 87 (2013) 012008 [arXiv:1208.0949] [INSPIRE].

[5] CMS collaboration, Search for supersymmetry at the LHC in events with jets and missing transverse energy, Phys. Rev. Lett. 107 (2011) 221804 [arXiv:1109.2352] [INSPIRE].

[6] L. Randall and R. Sundrum, Out of this world supersymmetry breaking, Nucl. Phys. B 557 (1999) 79 [hep-th/9810155] [INSPIRE].

[7] G.F. Giudice, M.A. Luty, H. Murayama and R. Rattazzi, Gaugino mass without singlets, JHEP 12 (1998) 027 [hep-ph/9810442] [INSPIRE].

[8] I. Jack and D.R.T. Jones, Fayet-Iliopoulos D terms and anomaly mediated supersymmetry breaking, Phys. Lett. B 482 (2000) 167 [hep-ph/0003081] [INSPIRE].

[9] E. Katz, Y. Shadmi and Y. Shirman, Heavy thresholds, slepton masses and the $\mu$ term in anomaly mediated supersymmetry breaking, JHEP 08 (1999) 015 [hep-ph/9906296] [INSPIRE].

[10] N. Arkani-Hamed, D.E. Kaplan, H. Murayama and Y. Nomura, Viable ultraviolet insensitive supersymmetry breaking, JHEP 02 (2001) 041 [hep-ph/0012103] [INSPIRE].

[11] R. Sundrum, 'Gaugomaly' mediated SUSY breaking and conformal sequestering, Phys. Rev. D 71 (2005) 085003 [hep-th/0406012] [INSPIRE].

[12] K. Hsieh and M.A. Luty, Mixed gauge and anomaly mediation from new physics at $10 \mathrm{TeV}$, JHEP 06 (2007) 062 [hep-ph/0604256] [INSPIRE].

[13] Y. Cai and M.A. Luty, Minimal gaugomaly mediation, JHEP 12 (2010) 037 [arXiv: 1008.2024$]$ [INSPIRE].

[14] T. Kobayashi, Y. Nakai and M. Sakai, (Extra)ordinary gauge/anomaly mediation, JHEP 06 (2011) 039 [arXiv:1103.4912] [INSPIRE].

[15] A. Pomarol and R. Rattazzi, Sparticle masses from the superconformal anomaly, JHEP 05 (1999) 013 [hep-ph/9903448] [INSPIRE].

[16] R. Rattazzi, A. Strumia and J.D. Wells, Phenomenology of deflected anomaly mediation, Nucl. Phys. B 576 (2000) 3 [hep-ph/9912390] [INSPIRE].

[17] F. Wang, Deflected anomaly mediated SUSY breaking scenario with general messenger-matter interactions, Phys. Lett. B 751 (2015) 402 [arXiv:1508.01299] [INSPIRE].

[18] N. Okada, Positively deflected anomaly mediation, Phys. Rev. D 65 (2002) 115009 [hep-ph/0202219] [INSPIRE].

[19] N. Okada and H.M. Tran, Positively deflected anomaly mediation in the light of the Higgs boson discovery, Phys. Rev. D 87 (2013) 035024 [arXiv:1212.1866] [INSPIRE].

[20] F. Wang, W. Wang, J.M. Yang and Y. Zhang, Heavy colored SUSY partners from deflected anomaly mediation, JHEP 07 (2015) 138 [arXiv: 1505.02785] [INSPIRE].

[21] R. Kitano and Y. Nomura, A solution to the supersymmetric fine-tuning problem within the MSSM, Phys. Lett. B 631 (2005) 58 [hep-ph/0509039] [INSPIRE].

[22] R. Kitano and Y. Nomura, Supersymmetry, naturalness and signatures at the LHC, Phys. Rev. D 73 (2006) 095004 [hep-ph/0602096] [INSPIRE]. 
[23] H. Baer, V. Barger, P. Huang and X. Tata, Natural supersymmetry: LHC, dark matter and ILC searches, JHEP 05 (2012) 109 [arXiv:1203.5539] [INSPIRE].

[24] J. Cao, C. Han, L. Wu, J.M. Yang and Y. Zhang, Probing natural SUSY from stop pair production at the LHC, JHEP 11 (2012) 039 [arXiv:1206.3865] [INSPIRE].

[25] H. Baer, V. Barger, P. Huang, A. Mustafayev and X. Tata, Radiative natural SUSY with a 125 GeV Higgs boson, Phys. Rev. Lett. 109 (2012) 161802 [arXiv:1207.3343] [INSPIRE].

[26] A. Mustafayev and X. Tata, Supersymmetry, naturalness and light higgsinos, Indian J. Phys. 88 (2014) 991 [arXiv:1404.1386] [INSPIRE].

[27] K.J. Bae, H. Baer, N. Nagata and H. Serce, Prospects for Higgs coupling measurements in SUSY with radiatively-driven naturalness, Phys. Rev. D 92 (2015) 035006 [arXiv: 1505.03541] [INSPIRE].

[28] X. Tata, Supersymmetry: aspirations and prospects, Phys. Scripta 90 (2015) 108001 [arXiv: 1506.07151] [INSPIRE].

[29] H. Baer, Supersymmetry and dark matter post LHC8: why we may expect both axion and WIMP detection, AIP Conf. Proc. 1604 (2014) 289 [arXiv:1310.1859] [InSPIRE].

[30] K.J. Bae, H. Baer, H. Serce and Y.-F. Zhang, Leptogenesis scenarios for natural SUSY with mixed axion-higgsino dark matter, JCAP 01 (2016) 012 [arXiv:1510.00724] [INSPIRE].

[31] K.J. Bae, H. Baer and H. Serce, Natural little hierarchy for SUSY from radiative breaking of the Peccei-Quinn symmetry, Phys. Rev. D 91 (2015) 015003 [arXiv:1410.7500] [InSPIRE].

[32] K.J. Bae, H. Baer, A. Lessa and H. Serce, Mixed axion-wino dark matter, Front. Phys. 3 (2015) 49 [arXiv:1502.07198] [INSPIRE].

[33] H. Baer, (Mainly) axion dark matter, arXiv:1510.07501 [INSPIRE].

[34] H. Baer, V. Barger, P. Huang, D. Mickelson, M. Padeffke-Kirkland and X. Tata, Natural SUSY with a bino- or wino-like LSP, Phys. Rev. D 91 (2015) 075005 [arXiv:1501.06357] [INSPIRE].

[35] Z. Chacko and E. Ponton, Yukawa deflected gauge mediation, Phys. Rev. D 66 (2002) 095004 [hep-ph/0112190] [INSPIRE].

[36] J.A. Evans and D. Shih, Surveying extended GMSB models with $m_{h}=125 \mathrm{GeV}$, JHEP 08 (2013) 093 [arXiv: 1303.0228] [INSPIRE].

[37] A. Albaid and K.S. Babu, Higgs boson of mass $125 \mathrm{GeV}$ in GMSB models with messenger-matter mixing, Phys. Rev. D 88 (2013) 055007 [arXiv:1207.1014] [INSPIRE].

[38] N. Craig, S. Knapen, D. Shih and Y. Zhao, A complete model of low-scale gauge mediation, JHEP 03 (2013) 154 [arXiv:1206.4086] [INSPIRE].

[39] Z. Kang, T. Li, T. Liu, C. Tong and J.M. Yang, A heavy SM-like Higgs and a light stop from Yukawa-deflected gauge mediation, Phys. Rev. D 86 (2012) 095020 [arXiv:1203.2336] [INSPIRE].

[40] P. Byakti and T.S. Ray, Burgeoning the Higgs mass to $125 \mathrm{GeV}$ through messenger-matter interactions in GMSB models, JHEP 05 (2013) 055 [arXiv:1301.7605] [INSPIRE].

[41] W. Fischler and W. Tangarife, Vector-like fields, messenger mixing and the Higgs mass in gauge mediation, JHEP 05 (2014) 151 [arXiv:1310.6369] [INSPIRE]. 
[42] R. Ding, T. Li, F. Staub and B. Zhu, Focus point supersymmetry in extended gauge mediation, JHEP 03 (2014) 130 [arXiv: 1312.5407] [INSPIRE].

[43] Planck collaboration, P.A.R. Ade et al., Planck 2013 results. XVI. Cosmological parameters, Astron. Astrophys. 571 (2014) A16 [arXiv:1303.5076] [INSPIRE].

[44] WMAP collaboration, J. Dunkley et al., Five-year Wilkinson Microwave Anisotropy Probe (WMAP) observations: likelihoods and parameters from the WMAP data, Astrophys. J. Suppl. 180 (2009) 306 [arXiv:0803.0586] [INSPIRE].

[45] LUX collaboration, D.S. Akerib et al., First results from the LUX dark matter experiment at the Sanford Underground Research Facility, Phys. Rev. Lett. 112 (2014) 091303 [arXiv: 1310.8214] [INSPIRE].

[46] XENON100 collaboration, E. Aprile et al., Dark matter results from 225 live days of XENON100 data, Phys. Rev. Lett. 109 (2012) 181301 [arXiv:1207.5988] [INSPIRE].

[47] LUX-ZEPLin collaboration, LZ dark matter experiment webpage, http://lz.lbl.gov/detector/.

[48] R. Barbieri and G.F. Giudice, Upper bounds on supersymmetric particle masses, Nucl. Phys. B 306 (1988) 63 [inSPIRE].

[49] H. Baer, V. Barger and D. Mickelson, How conventional measures overestimate electroweak fine-tuning in supersymmetric theory, Phys. Rev. D 88 (2013) 095013 [arXiv:1309.2984] [INSPIRE].

[50] H. Baer, V. Barger, D. Mickelson and M. Padeffke-Kirkland, SUSY models under siege: LHC constraints and electroweak fine-tuning, Phys. Rev. D 89 (2014) 115019 [arXiv:1404.2277] [INSPIRE]. 